\title{
Comments on Hsu et al.: Surgical treatment of syndesmotic diastasis: emphasis on effect of syndesmotic screw on ankle function
}

\author{
Wouter J. van Zuuren • Jore H. Willems • \\ Michel P. J. van den Bekerom
}

Received: 31 December 2010 / Accepted: 13 January 2011 /Published online: 1 February 2011

(C) Springer-Verlag 2011

\section{Dear Editor,}

With great interest we read the article of Hsu et al. entitled "Surgical treatment of syndesmotic diastasis: emphasis on effect of syndesmotic screw on ankle function" [1]. The authors report on screw fixation in patients with syndesmotic diastasis. They describe a syndesmotic diastasis recurrence in six of 52 patients $(11.5 \%)$ with an average increase in widening of $3.8 \mathrm{~mm}$ (range 3-7 mm) diagnosed on anteroposterior radiographs. At 19 months (range 12-36) follow-up they observed no difference in ankle function, as defined by Bray's scoring system, between patients with and without recurrence of syndesmotic diastasis. We were surprised by their conclusion and would like to make some remarks that we feel should be discussed.

Distal tibiofibular syndesmotic injuries occur in over $10 \%$ of ankle fractures [2]. Syndesmotic instability is most commonly associated with pronation external rotation, pronation abduction or less frequently with supination external rotation trauma mechanisms. Syndesmotic instability leads to mortise widening and this should be treated with syndesmotic stabilisation to prevent long-term complications. Widening of the ankle mortise leads to increased stress on the articular cartilage. This statement is based on the studies of Ramsey and Hamilton, and later of Lloyd et al. When the talus moves $1 \mathrm{~mm}$ laterally, the contact area in the tibiotalar articulation is decreased by $42 \%$. This results in an increase in the stress per unit area on the articular cartilage $[3,4]$.

Leeds and Ehrlich found a negative correlation between syndesmosis instability and subjective outcome, objective

W. J. van Zuuren · J. H. Willems $(\bowtie) \cdot$ M. P. J. van den Bekerom Department of Orthopaedic Surgery, Spaarne Hospital, Spaarnepoort 1, P.O. Box 770, 2134 Hoofddorp, The Netherlands e-mail: jorewillems@gmail.com outcome and degenerative changes in the ankle joint at four years follow-up [5]. Pettrone et al. concluded that if the syndesmosis is not adequately reduced the function scores were significantly lower after one to five years follow-up [6].

We think that there are some potential reasons why the authors came to their conclusions.

1. As already stated by the authors, a larger sample size could possibly provide significance. In this series six patients out of a total of 52 sustained recurrence of syndesmotic widening.

2. There are many tests available to evaluate ankle function. The authors have used the Bray's scoring system. No validity studies or methodological evaluation have been performed proving this system to be adequate for scoring ankle function [7].

3. The criteria used for recurrence of syndesmotic diastasis are controversial. Beumer et al. stated that no optimal radiographic parameter exists to assess syndesmotic integrity, because all are dependent on the position of rotation. Except for unilateral absence of tibiofibular overlap and the combination of the medial and superior clear space, quantitative measurement of syndesmotic parameters in repeated ankle radiographs may be of little value [8].

4. The most important cause could be the short follow-up. It is likely that degenerative osteoarthritis was already developing but, in this short follow-up, had not yet resulted in reduced ankle function. It would be interesting to increase the follow-up or check the status of the tibiotalar joint with X-ray images to examine early degenerative pathology.

We are of the opinion that recurrent syndesmotic widening leads to long-term degenerative changes. Early treatment is 
necessary because late repairs give satisfactory but less favourable outcomes in comparison to properly treated acute injuries. It is not easy to regain complete stability by means of these secondary procedures [9]. We agree with the authors that more research is necessary to confirm the reliability and validity of the results presented. We also hope the authors will present the long-term outcome of this series.

\section{References}

1. Hsu YT, Wu CC, Lee W, Fan KF, Tseng IC, Lee PC (2010) Surgical treatment of syndesmotic diastasis: emphasis on effect of syndesmotic screw on ankle function. Int Orthop. doi:10.1007/ s00264-010-1147-9

2. Court-Brown CM, McBirnie J, Wilson G (1998) Adult ankle fractures: an increasing problem? Acta Orthop Scand 69:43-47
3. Ramsey PL, Hamilton W (1976) Changes in tibiotalar area of contact caused by lateral talar shift. J Bone Joint Surg Am 58(3):356-357

4. Lloyd J, Elsayed S, Hariharan K, Tanaka H (2006) Revisiting the concept of talar shift in ankle fractures. Foot Ankle Int 27(10):793-796

5. Leeds HC, Ehrlich MG (1984) Instability of the distal tibiofibular syndesmosis after bimalleolar and trimalleolar ankle fractures. J Bone Joint Surg Am 66:490-503

6. Pettrone FA, Gail M, Pee D, Fitzpatrick T, van Herpe LB (1983) Quantitative criteria for prediction of the results after displaced fracture of the ankle. J Bone Joint Surg Am 65:667-677

7. Suk M, Hanson BP, Norvell DC, Helfet DL (2009) Bray ankle evaluation score. In: Suk M, Hanson BP, Norvell DC, Helfet DL (eds) Musculoskeletal outcomes measures and instruments. AO publishing, Davos, pp 648-649

8. Beumer A, van Hemert WL, Niesing R, Entius CA, Ginai AZ, Mulder PG, Swierstra BA (2004) Radiographic measurement of the distal tibiofibular syndesmosis has limited use. Clin Orthop Relat Res 423:227-234

9. van den Bekerom MP, de Leeuw PA, van Dijk CN (2009) Delayed operative treatment of syndesmotic instability. Current concepts review. Injury 40(11):1137-1142 